\title{
Microstructural Simulation of Grain Growth in Two-phase Polycrystalline Materials
}

\author{
${ }^{*}$ R. El-Khozondar', H. El-Khozondar', G. Gottstein ${ }^{3}$, A. Rollet ${ }^{4}$ \\ ${ }^{1}$ Department of Physics, Al-Aqsa University, Gaza, Palestine \\ ${ }^{2}$ Electrical and Computer Engineering Dept., Islamic Univ., \\ Gaza, Palestine \\ ${ }^{3}$ Institut für Metallkunde und Metallphysik, RWTH Aachen, Germany \\ ${ }^{4}$ Department of Material Sci. \& Eng., CMU, Pittsburgh, PA, U. S. A.
}

A quantity of vital engineering materials is example of two-phase materials in which each phase may be polycrystalline. In numerous cases, both phases of these materials may undergo grain growth which may affect their electrical and mechanical properties. Therefor, substantial efforts have been devoted to understand the microstructural evolution of polycrystals to improve their performance in microelectronic industries. Numerical method based on Monte Carlo Potts model is used to investigate microstructural evolution of two-phase polycrystalline materials in which grain growth in both cases is controlled by grain boundary diffusion $(n=4)$. It is shown that the microstructural evolution of two-phase polycrystals eventually reaches an asymptotic regime in which grain growth in both phases is coupled due to Zener pinning and obeys a power-law relationship $d \sim t^{1 / n}$. This conclusion is valid in a broad parameter range and is compatible with theoretical predictions and laboratory experiments.

\section{Introduction:}

Most important metallic and ceramic materials are polycrystalline. They consist of small grains which are glued together by interatomic forces. When polycrystals are heated, they experience different changes with time, which governs the mechanical, thermal and electrical properties of the materials. Therefore, it is a critical issue to understand the process of grain growth in engineering materials.

\footnotetext{
*rifa20012002@yahoo.com
} 
Grain growth is a process in which grain size of single-phase polycrystalline material increases with time, driven by the reduction in the total grain boundary energy. The value of the grain growth exponent for pure metals and ceramics is 2 (Beck et al., 1948; Burke, 1949; Burke and turnbull, 1952). It can be obtained with the assumption that the energy difference across curved grain boundaries $\Delta \mathrm{G}$ (the driving force) is inversely proportional to the grain size $d$. Assuming that the rate of change of the grain size is proportional to the driving force $\Delta \mathrm{G}$, we get $\mathrm{d} \sim \mathrm{t}^{1 / 2}$ which can be written in more general form

$$
\mathrm{d}=\mathrm{kt}^{1 / \mathrm{n}}
$$

where $\mathrm{n}$ is the grain growth exponent and $\mathrm{k}$ is the grain growth constant. Since the velocity of grain boundary is controlled by the rate at which atoms jump across the boundary, the typical diffusion distance for atoms associated with grain boundary migration is on the order of the boundary width which is about 2 or 3 lattice parameters for a pure metal. Experimentally determined values of grain growth exponent for a variety of metals and ceramics vary from $n \sim 2$ to 4 (Anderson et al., 1984).

On the other hand, Ostwald ripening is defined as the process during which large second-phase particles grow while small particles reduce in size and disappear. Thus, the interfacial energy between the matrix and the second phase is reduced. The driving force is the difference in the chemical potential of atoms in small and large particles of the second phase. Atoms detached from small particles of the second phase must diffuse through the matrix to attach to large particles of the second phase so that large grains grow at the expense of small particles. Consequently, the typical diffusion distance for atoms is on the order of the partition distance between particles of the second phase. The grain growth exponent for Ostwald ripening of particles in polycrystalline materials is $n=3$ if grain growth is controlled by volume diffusion (Lifshitz and Slyozov, 1961; Wagner, 1961), $n=4$ in case of grain boundary diffusion controlled grain growth and $n=5$ for diffusion on dislocations (Ardell, 1972). If grain growth is controlled by surface nucleation then Ostwald ripening obeys a logarithmic rather than power-law function of time (Solomatov and Stevanson, 1993). In this case the value of $\mathrm{n}$ can be random large.

Grain growth and Ostwald ripening may happen concurrently, especially, in multiphase polycrystalline materials. These materials such as ceramics and metallic alloys have important engineering applications. Particular examples are the $\mathrm{ZrO}_{2}-\mathrm{Al}_{2} \mathrm{O}_{3}$ two-phase in ceramics (French et al., 1990; Lang et al., 1987; Alexander et al., 1994) and the near eutectic solder alloys which are commonly used in electrical, mechanical and thermal 
interconnects between an integrated chip or component and a substrate or printed circuit board (Satoh,1993). If the second-phase particles were stable, grain growth in the major phase would stop as soon as the grain size reaches some maximum value which is proportional to the second phase grain size (Smith, 1948). If the second-phase particles undergo Ostwald ripening, the grains of the major phase can grow too. Hillert (1965) and Gladman (1966) proposed that after an adequately extended time, grain growth in two-phase systems in which both phases grow is controlled by Ostwald ripening of the dispersed particles and is coupled due to Zener pinning. There is experimental proof of such coupled growth (Mader, 1965; Holm et al., 1977, Garwel and Ankem, 1989, 1990a, 1990b; Higgins, 1992; Ankem, 1992, Alexander,194) and in numerical simulations (Fan, 1997, 1998; Chen, 1996, Solomatov et al., 2002).The grain growth exponent for both phases is similar and corresponds to one of the mechanisms of Ostwald ripening.

The main purpose of this paper is to systematically study the kinetics of grain growth and Ostwald ripening in two-phase polycrystalline materials by employing computer simulations based on the Monte Carlo Potts approach. In these systems, the grain boundary migration of the major phase is pinned by the second phase particles which coarsen through long range diffusion. Based on the computer simulation we will determine the grain growth exponent and the grain growth constant. Additionally, the effect of Zener pinning on grain growth of the major with growing second-phase particles will be determined, in a term that, after sufficiently long time the ratio of the major phase grain size to the second phase grain size will be constant whose value depends on the volume fraction of the second phase.

\section{Simulation Methodology:}

In this study, numerical method based on the Monte Carlo Potts model is used to investigate grain growth in two-phase polycrystalline materials. It was developed more than four decades ago as an extension to the ferromagnetic Ising model for magnetic domain evolution (Potts, 1952). The Ising model (1925) represents a magnetized material as a collection of spins where only two states are possible, namely up or down. Potts later generalized the Ising model and allowed for Q states for each particle in the system. Although this model has been developed a long time ago, not until much later, it was very successful in describing the grain growth in polycrystals. The reason for this is a similarity between the ferromagnetic Potts structures and grain structures; both are being characterized by an array of cells having the same spin or crystal lattice orientation. These cells are separated by boundaries which evolve in such a way as to minimize total interfacial energy. 
The two-phase polycrystalline material is treated as two-dimensional triangular array of sites. Each site is assigned a spin, $\mathrm{q}_{\mathrm{i}}$, which corresponds to the orientation of the grain in which it is embedded. Lattice sites that are adjacent to sites having different grain orientations are regarded as being separated by a grain boundary, whereas a site surrounded by sites with the same orientation is in the grain interior. The sites that correspond to the major phase (phase A) are given positive numbers. The sites correspond to the second phase (phase B) are given negative numbers.

The total system energy is dependent only on the spin exchange energy which can be written as follows:

$$
E=\frac{1}{2} \sum_{i=1}^{N} \sum_{j=1}^{6}\left[1-\delta\left(q_{i}, q_{j}\right)\right]
$$

where $\mathrm{N}$ is the total number of sites, $\delta$ is the Kronecker delta function and $\mathrm{q}_{\mathrm{i}}$ is the spin of $i$ th site. The only energy considered in the simulation is the interfacial energy; therefore, for any pair of sites, different spins result in an interfacial energy of unity and same pins result in an interfacial energy of zero.

The microstructure evolved as a result of grain growth boundary migration and long-range diffusion. The grain boundary migration is simulated by picking a site and a new orientation at random from the set of allowable values. The change in total system energy $\Delta \mathrm{E}$ for reorienting the site is computed, and the reorientation is implemented with the transition probability,

$$
\mathrm{P}= \begin{cases}1, & \Delta \mathrm{E} \leq 0 \\ 0, & \Delta \mathrm{E} \geq 0 .\end{cases}
$$

The long-range diffusion is simulated by spin exchange between the phases. A site and its neighbor are selected at random. If the two sites belong to different phases, they are allowed to exchange their spins. The change in total system energy $\Delta \mathrm{E}$ for the spin exchange is calculated, and the spin exchange is determined by using the full Arrhenius function,

$$
\mathrm{P}= \begin{cases}1, & \Delta \mathrm{E} \leq 0 \\ \mathrm{e}^{-\frac{\Delta \mathrm{E}}{\mathrm{k}_{\mathrm{B}} \mathrm{T}}}, & \Delta \mathrm{E} \geq 0 .\end{cases}
$$

where $k_{B}$ is the Boltzman constant and $T$ is the temperature. If the energy change is negative or zero the move is accepted. If the energy change is positive, a random number $R$ between 0 and 1 is generated such that if $R \leq P$ the change will be accepted. 
One Monte Carlo time step (1 MCS) is typically defined as $\mathrm{N}$ reorientation attempts, i.e. each site is given an opportunity to change orientation. The number of Monte Carlo Steps is assumed to be proportional to physical time. Time is incremented after each attempt spin flip by 1/N MCS. The temperature for spin exchange is $\mathrm{T}=0.7$. The reason for choosing this value is to low-temperature regimes which gives low-grain growth exponents.

The structure of a two-dimensional two-phase polycrystalline material is represented with the help of a $400 \mathrm{X} 400$ triangular lattice. Each site represents a group of atoms with a particular spin. Although in reality, the spins range is continuous, the results do not depend on the values of spin, Q (Anderson et al., 1984; Sahni et al., 1983; Grest et al., 1988). The value Q=100 is adequately large to eliminate the effect of coalescence; therefore, it is used in all our simulations. The controlling parameters of the two phases are chosen to be identical. This means that the case with a volume fraction of $f_{v}$ of the second phase is equivalent to one with a volume fraction $\left(1-f_{v}\right)$ of the major phase and vice versa. Various volume fractions of the second phase are considered.

\section{Simulation Results:}

\subsection{Grain growth kinetics in two-phase systems}

Microstructural evolution corresponding to grain growth in two-phase polycrystalline materials with volume fractions of phase B varies from $10 \%$ to $50 \%$ is displayed in Fig. 1. The fraction 0\% B in Fig. 1 represents grain growth in one-phase polycrystals for comparison. In these microstructures, the grains of phase A are white and the grains of phase B are gray. Grain growth kinetics is described by Eq. (1). The slope of a straight fit through this $\log d$ versus $\log t$ plot by least-square fitting is the inverse of grain growth exponent, $1 / \mathrm{n}$. The average grain size is drawn as a function of time in Fig. 2, with various volume fractions, $f_{v}$, in the one-phase material, $f_{v}=0$. As shown in Fig. 2, the grain size initially evolves pretty slowly but eventually reaches the asymptotic regime consistent with $d \alpha t^{1 / n}$. The early time transient is due to the initial conditions. In fitting the data shown in Fig. 2 to determine the grain growth kinetics, we found that the results were sensitive to the time regime examined.

When all the data for time $t>10000$ MCS was included in the least square fit to Eq. (1), the grain growth exponent and the rate constant obtained for various $f_{v}$ are shown in Fig. 3 and Fig. 4. As can be seen from Fig. 3, in onephase system, the inverse of grain growth exponent $1 / \mathrm{n}$ is close to 0.5 in agreement with previous simulation results (Anderson et al., 1984; Sahni et al., 1983; Grest et al., 1988). In two-phase systems the value of $1 / \mathrm{n}$ for phase B is close to $1 / 4$ estimated for grain-boundary diffusion controlled grain growth. For Phase $\mathrm{A}$, the value of $1 / \mathrm{n}$ goes higher than $1 / \mathrm{n}$ as the volume fraction increases. 
The reason for this is that the grains of phase A are interconnected and grow via grain growth; in addition, to long range diffusion via grain boundary migration. As shown in Fig. 4, the rate constant $\mathrm{k}$ for phase B is higher than the rate constant for phase $\mathrm{A}$. As the volume fraction of phase $\mathrm{B}$ increases the value of $\mathrm{k}$ increases, while the value of $\mathrm{k}$ decreases as the volume fraction of phase $\mathrm{A}$ increases. The ratio of the rate constant of phase $\mathrm{A}\left(\mathrm{k}_{\mathrm{A}}\right)$ is to the rate constant of phase $B\left(k_{B}\right)$ is drawn as a function of volume fraction $f_{v}$ in Fig. 5. As can be seen from Fig. 5, the ratio $k_{A} / k_{B}$ increases linearly as the volume fraction of phase $\mathrm{B}$ increases and decreases linearly as the volume fraction of phase A increases. This relation can be expressed as
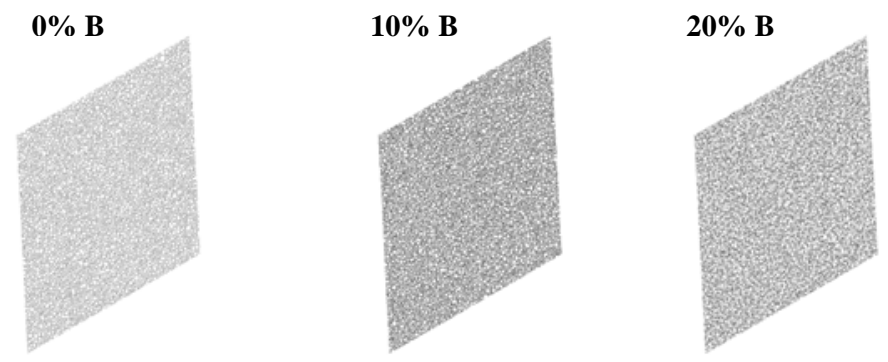

\section{$\mathrm{t}=1000 \mathrm{MCS}$}
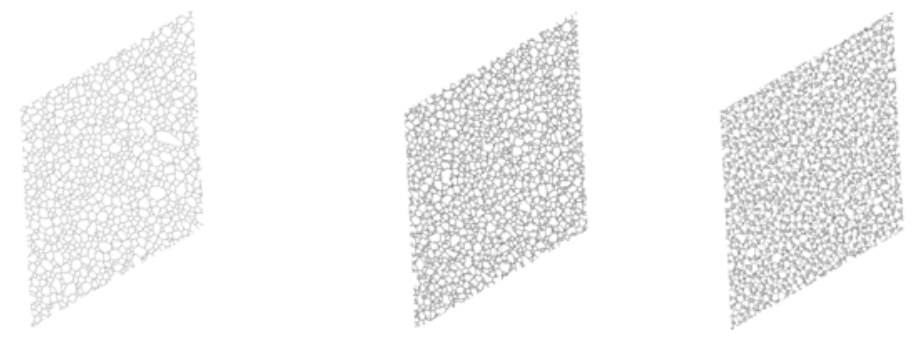

$\mathbf{t}=10000 \mathrm{MCS}$
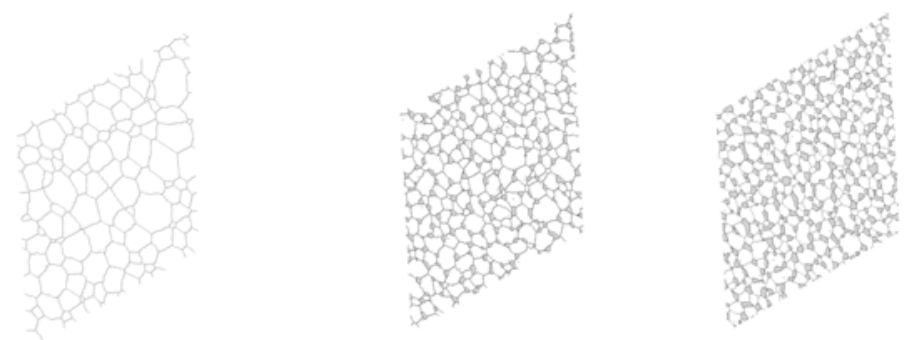

$\mathrm{t}=100000 \mathrm{MCS}$

Fig. (1): Microstructural evolution in a two-phase system having different fractions of the second phase, B. The fraction $0 \% \mathrm{~B}$ indicates grain growth in one-phase system. System size is $400 \mathrm{X} 400$ triangular lattices. Phase A grains are white and phase B grains are gray. 

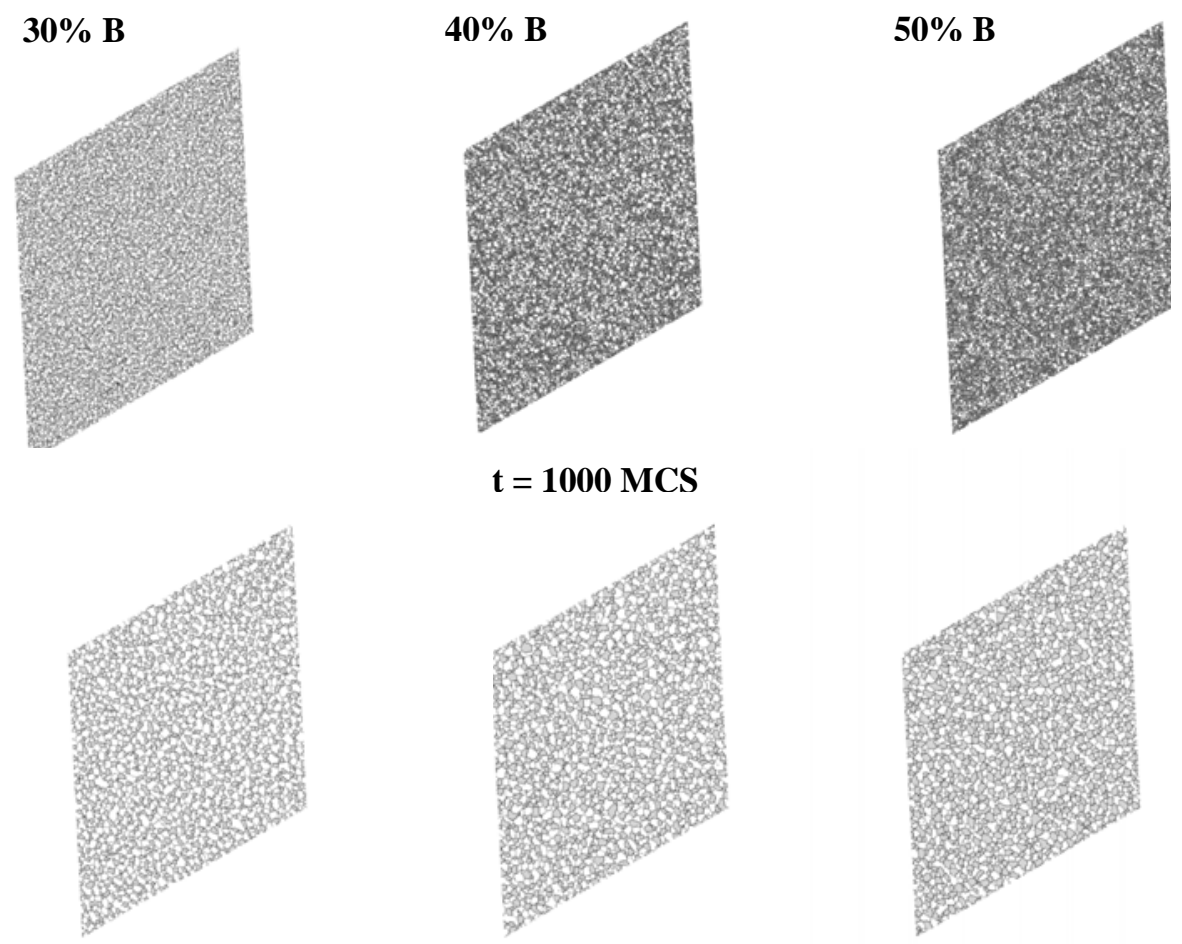

$$
t=1000 \mathrm{MCS}
$$
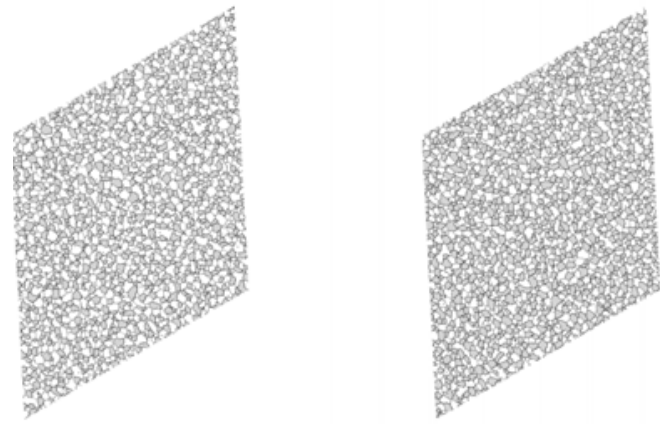

$$
\mathrm{t}=10000 \mathrm{MCS}
$$
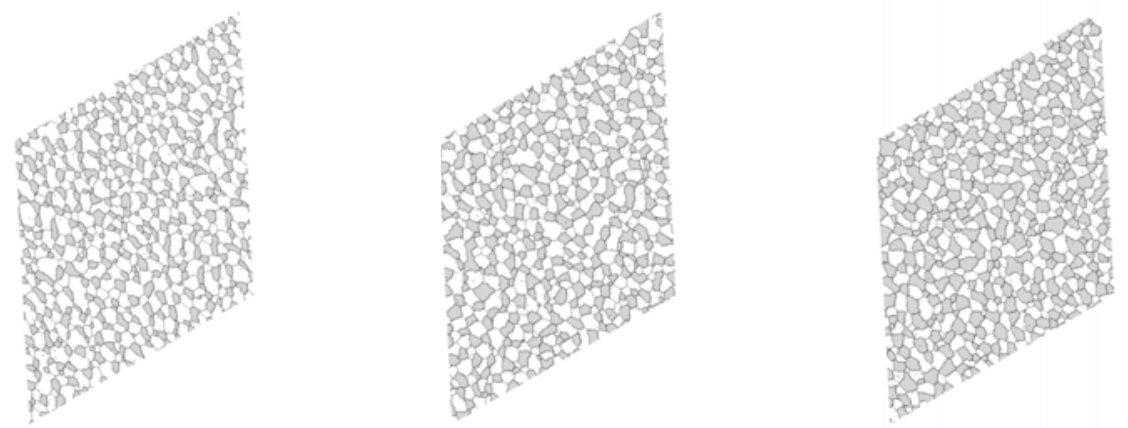

\section{$\mathbf{t}=100000 \mathrm{MCS}$}

Fig. (1): (Continued) 

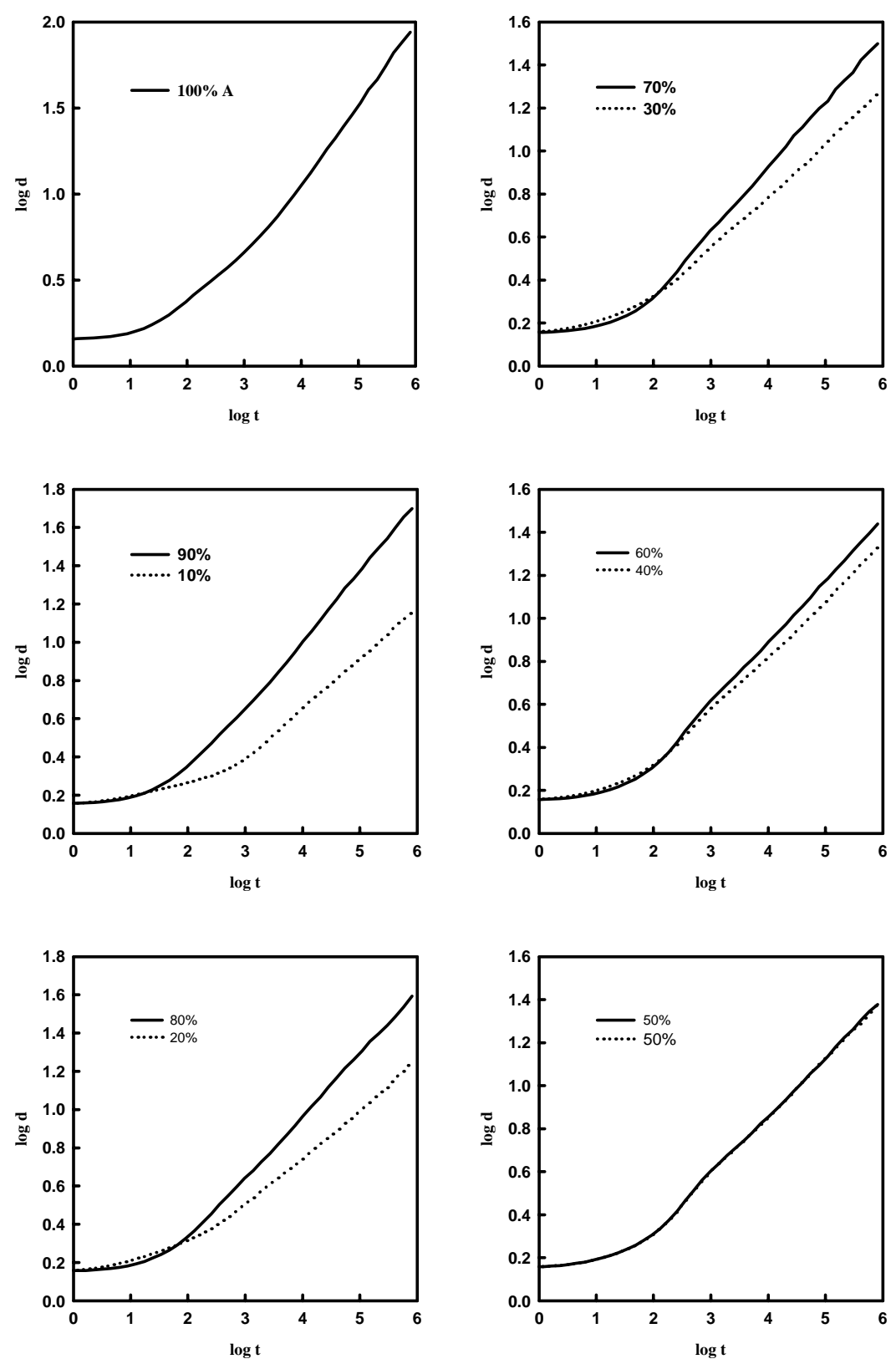

Fig. (2): Time dependence of the mean grain size for grain growth simulation in twophase systems. The ratio of each phase is indicted. The fraction 100\% A corresponds to grain growth in one-phase system. 


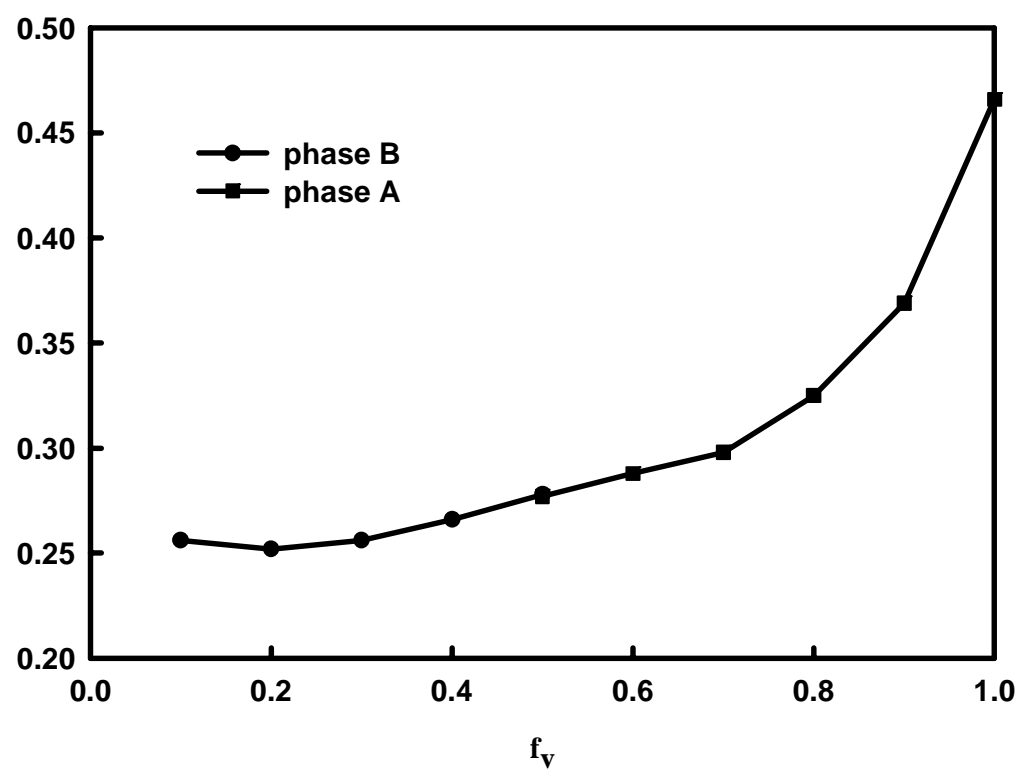

Fig. (3): The inverse of grain growth exponent, $1 / n$, as a function of volume fraction, $f_{v}$.

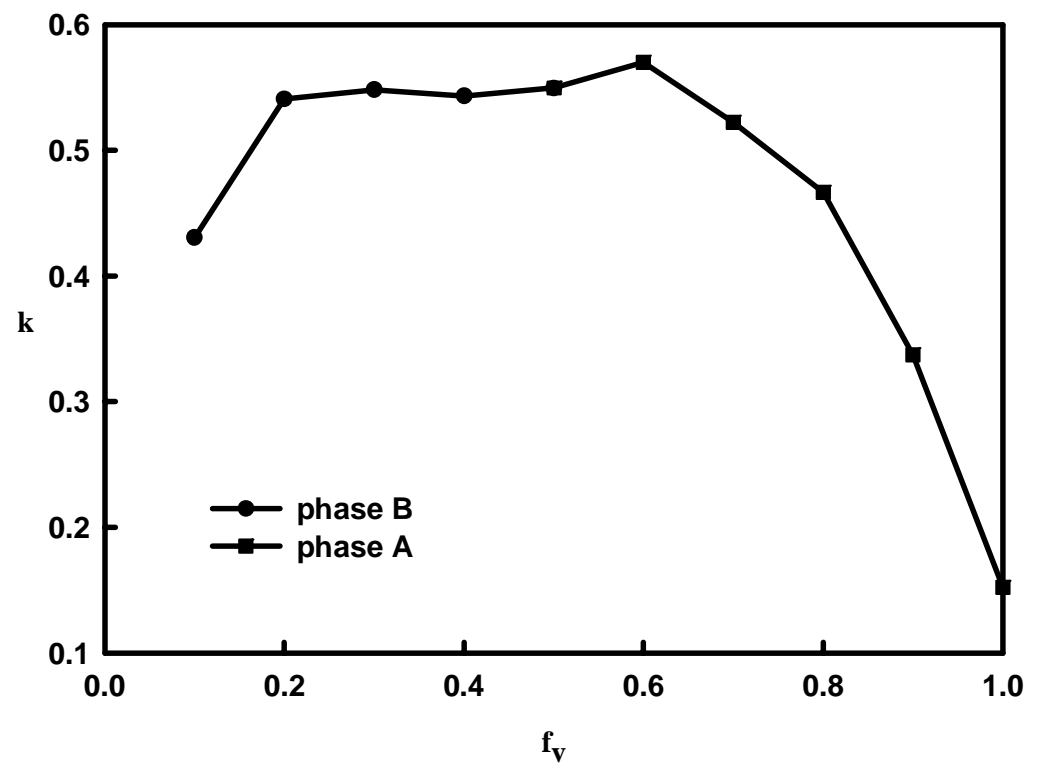

Fig. (4): The rate constant, $k$ of grain growth as a function of volume fraction, $f_{v}$. 


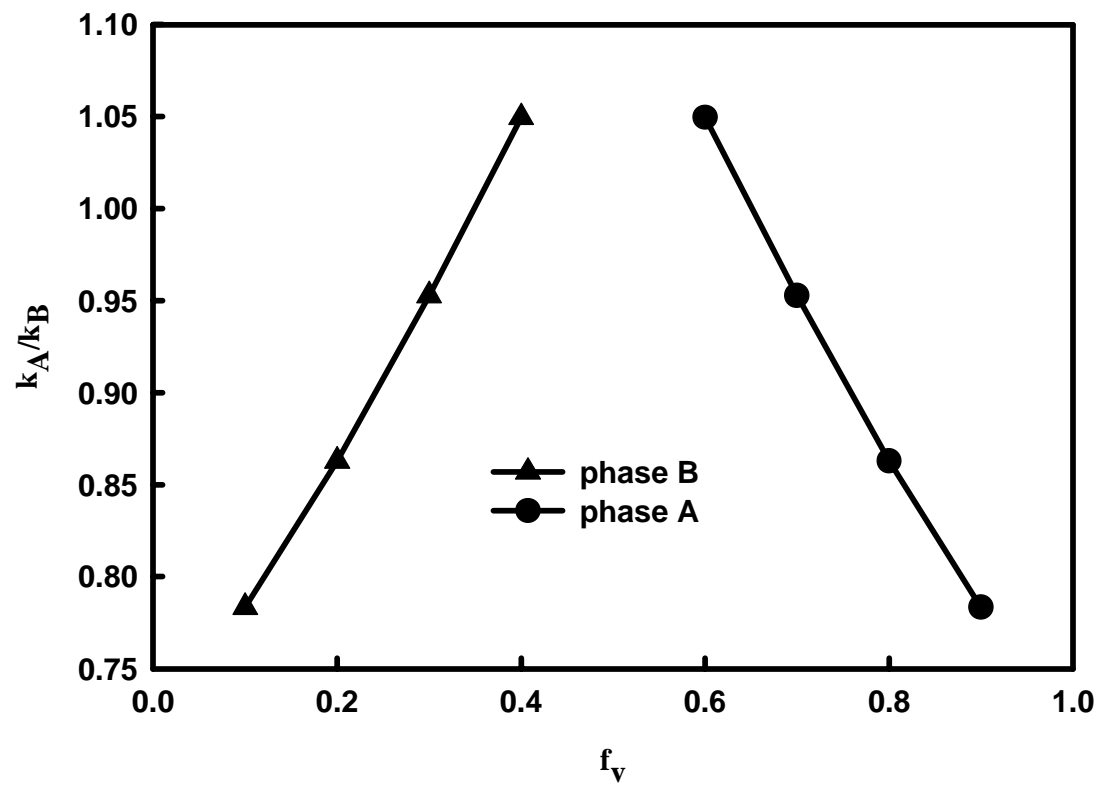

Fig. (5): Ratio of the rate constant of phase $A\left(k_{A}\right)$ to the rate constant of phase $B\left(k_{B}\right)$ versus volume fraction $f_{v}$.

$$
\mathrm{k}_{\mathrm{A}}=\frac{\mathrm{k}_{\mathrm{B}}}{\mathrm{f}_{\mathrm{A}}^{\mathrm{n}_{\mathrm{A}}}}
$$

where $\mathrm{f}_{\mathrm{A}}$ is the volume fraction of phase $\mathrm{A}$ and $\mathrm{n}_{\mathrm{A}}$ is an impedance exponent depends on the volume fraction. This relation is consistent with the experimental results (Grewel and Ankem, 1989; 1990).

\subsection{Zener pinning with growing second-phase particles}

Zener pinning is a phenomenon in which second-phase particles impede the grain growth of the major phase by pinning the motion of grain boundaries. The pinned growth state occurs when the grains of the major phase would reach limited size which is proportional to the size of the second-phase particles. The relationship between the limited grain size and the second phase parameters can be expressed as 


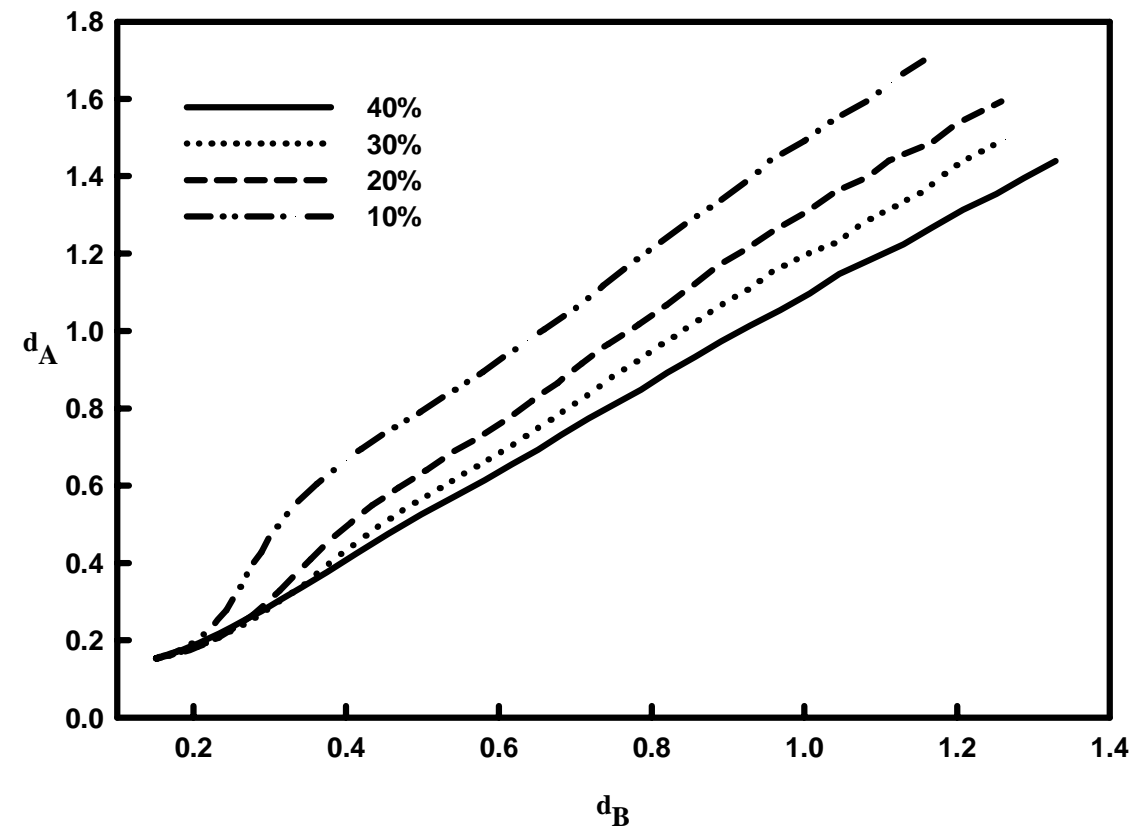

Fig. (6): The grain size of phase A versus the grain size of phase B. The fraction of the second phase is shown in the figure.

$$
\mathrm{d}_{\mathrm{A}}=\frac{\mathrm{c}}{\mathrm{f}_{\mathrm{v}}^{\mathrm{m}}} \mathrm{d}_{\mathrm{B}}
$$

where $d_{A}$ is the grain size of the major phase, $d_{B}$ is the grain size of the secondphase, $\mathrm{c}$ and $\mathrm{m}$ are constants. The value $\mathrm{m}=1$ was considered by the early Zener model (Smith, 1948). Under certain conditions, it was proposed that the value $\mathrm{m}=1 / 2$ for nonrandom, two-dimensional systems (Srolovitz et al., 1984; Doherty, 1987) and $m=1 / 3$ for high-volume fractions, three-dimensional systems (Hellman and hillert, 1975; Hillert, 1988; Hassold et al., 1990).

The present simulation result of the relationship between $d_{A}, d_{B}$ and $f_{v}$ is shown in Fig. 5. The average grain size of phase $A\left(d_{A}\right)$ depends linearly on the grain size of the second phase $B\left(d_{B}\right)$ for all volume fractions of the second phase from $10 \%$ to $40 \%$ which agrees well with experimental results (Alexander et al., 1994). 


\section{Conclusion:}

The kinetics of grain growth and Ostwald ripening in two-phase polycrystalline materials have been studied using computer simulations based on Monte Carlo Potts model. The same model have been used to investigate Zener pinning in the presence of growing second-phase particles. The simulation results show that the growth kinetics of both phases are controlled by long rang diffusion and follow the power-growth law Eq. (1) with $n=4$. The rate constant of phase $\mathrm{A}\left(\mathrm{k}_{\mathrm{A}}\right)$ varies linearly with the rate constant of phase $\mathrm{B}$ $\left(\mathrm{k}_{\mathrm{B}}\right)$. The remarkable variation comes from the different diffusion distances of the two phases during growth as the volume fraction changes. For the low volume fraction of phase B, the growth kinetics are controlled by ostwald ripening and the typical diffusion distance is on the order of the typical distance between B-phase grains. However, for the high volume fraction of A-phase, the grain growth kinetics depends on the grain boundary width that is pinned by $\mathrm{B}$-phase grains and the volume fraction of phase $\mathrm{B}$. A linear relation between A-phase grain size $\left(d_{A}\right)$ and B-phase grain size $\left(d_{A}\right)$ is found for all volume fractions of phase $\mathrm{B}$ which are compatible with experimental results. In conclusion, our simulation results present an independent support for the Hillert (1965) and Gladman's (1966) proposal that microstructural evolution of twophase systems ultimately reaches an asymptotic regime in which grain growth in both phase is coupled due to Zener pinning and is controlled by long range diffusion.

\section{Refernces}

1. P. A. Beck, J. C. Kremer, L. J. Dremer and M. L. Holzworth, Trans. AIME 175, 372-394 (1948).

2. J. E. Burke, Trans. Metall. Soc. AIME 180, 73-91 (1949).

3. J. E. Burke and D. Turnbull, Prog. Metal. Phys. 3, 220-292 (1952).

4. M. P. Anderson, D. J. Srolovitz, G. S. Grest and P. S. Sahni, Acta Metall. 32, 783-971 (1984).

5. I. M. Lifshitz and V. V. Slyozov, J. Phys. Chem. Solids 19, 35-50 (1961).

6. C. Wagner, Electrochem. 65, 581-591 (1961).

7. A. J. Ardell, Acta Metall. 20, 601-609 (1972).

8. S. V. Solomatov and D. J. Stevanson, J. Geophys. Res. 98, 5407-5418 (1993).

9. J. D. French, M. P. Harmer, H. M Chan and G. A. Miller, J. Am. Ceram. Soc. 73, 2508-2510 (1990).

10. F. F. Lange and M. M. Hirlinger, J. Am. Ceram. Soc. 70, 827-833 (1987). 
11. K. B. Alexander, P. F. Becher, S. B. Waters and A. Bleier, J. Am. Ceram. Soc. 77, 939-946 (1994).

12. R. Satoh, "Thermal Stress and Strain in microelectronics packaging," $A$ Prediction of the Thermal Fatigue Life of Solder Joints Using Crack Propagation Rate and Equivalent Strain Range, ed. J.H. Lau (Van Nostrand Reinhold, 1993) pp. 501-531.

13. S. Smith, Trans. AIME 175, 15-51 (1948).

14. M. Hillert, Acta Metall. 13, 227-238 (1965).

15. T. Gladman, Proc. R. Soc. London 294A, 298-309 (1966).

16. K. Mäder and E. Hornbogen, Scripta Met. 8, 979-984 (1974).

17. K. Holm, J. D. Embury and G. R. Purdy, Acta Metall. 25, 1191-1200 (1977).

18. G. Grewal and S. Ankem, Metall. Trans. 20A, 38-54 (1989).

19. G. Grewal and S. Ankem, Metall. Trans. 21A, 1645-1654 (1990a).

20. G. Grewal and S. Ankem, Acta Metall. 38, 1607-1617 (1990b).

21. G. T. Higgins, S. Wuryolukito and P. Nash, Mater. Sc. Forum 92-96, 671676 (1992).

22. S. Ankem, Mater. Sc. Forum 92-96, 159-168 (1992).

23. K. B. Alexaner, P. F. Becher, S. B. Waters and A. Bleier, J. Am. Ceram. Soc. 77, 939-946 (1994).

24. D. Fan and L. Q. Chen, J. Am. Ceram. Soc. 80, 1773-1780 (1997).

25. D. Fan, L. Q. Chen and S. P. P. Chen, J. Am. Ceram. Soc. 81, 526-532 (1998).

26. L. Chen and D. Fan, J. Am. Ceram. Soc. 79, 1163-1168 (1996).

27. V. S. Solomatov, R. El-Khozondar, V. Tikare, Phys. Earth Planet. Inter. 129, 265-282 (2002).

28. R. B. Potts, Proc. Camb. Phil. Soc. 48, 106-109 (1952).

29. E. Ising, Zeitschrift für Physik 31, 253-258 (1925).

30. P. S. Sahni, J. D. Srolovitz, G. S. Grest, M. P. Anderson, S. A. Safran, Phys. Rev. 28B, 2705-2716 (1983).

31. G. S. Grest, M. P. Anderson, D. J. Srolovitz, Phys. Rev. 38B, 4752-4760 (1988).

32. D. J. Srolovitz, M. P. Anderson, G. S. Grest and P. S. Sahni, Acta Metal. 32, 1429-38 (1984).

33. R. D. Doherty, D. J. Srolovitz, A. D. Rollet and M. P. Anderson, Scr. Metall. 21, 675 (1987).

34. P. Hellman and M. Hillert, Scan. J. Metall. 4, 211 (1975).

35. M. Hillert, Acta Metal. 36, 3177-81 (1988).

36. G. N. Hassold, E. A. Holm and D. J. Srolovitz, Scr. Metall. 24, 101-106 (1990). 